\title{
ARM Processor Based Wireless Data Acquisition System for Pressure Distribution Analysis
}

\author{
Ms. Pavithra $\mathrm{M}^{1}$, Ms. Jayanthi $\mathrm{K}^{2}$, Ms.Kanimozhi $\mathrm{R}^{3}$, Ms.Priya $\mathrm{N}^{4}$ and Mr.Ramesh. $\mathrm{K}^{5}$ \\ ${ }_{1,2,3,4} \mathrm{UG}$ students Department of Electronics and Communication Engineering, V.R.S.College of Engg \& Tech, Villupuram, \\ Tamil Nadu, India
}

${ }^{5}$ Assistant Professor, Department of Electronics and Communication Engineering, V.R.S.College of Engg \& Tech, Villupuram, Tamil Nadu, India

\begin{abstract}
Techniques that could precisely monitor human motion are use ful in applications such as rehabilitation, virtual re ality, sports science, and surveillance. Pressure distributions in the human feet are important and use ful me as ures in footwe ar evaluation, athle tic training, clinical gait analys is, and pathology foot diagnosis. Most of the existing $\mathrm{s}$ ys te $\mathrm{ms}$ re quire wiring that res trains the natural move me nt and are also uncomfortable to we ar. Comme rcially available systems are too expensive for small establishments. To ove rcome these limitations, a we arable wire less sens or ne twork using low-cost, low-power wire less sensor platform imple mented using an IEEE 802.15.4 wire less standard can be de veloped with compact we arable sensors. An e mbedded syste m using ARM processor has been considered for the hardware and to gain access to laptop, which can be configured to comfortably work in rese arch laboratories, clinics, sport ground, and other outdoor environments. A method by which an interactive front end can be de veloped for this application has been discussed. The results are displaye d as values by ins tantane ous signals and pie charts. Also provision has been made to calculate and dis play peak press ure and mean pressure at a given point.
\end{abstract}

Keywords: Foot pressure measurement, wireless sensor network, biomedical application, embedded syste $\mathrm{m}$, ARM processor.

\section{INTRODUCT ION}

Measurement of footpressure that is present in the human foot is called plantar pressure measurement which is an useful parameter which can be effectively used in various commercial and medical applications. At the first instant it can has the commercial application of evaluation of footwear. It can be used to evaluate the effectiveness of therapeutic and athletic shoes with and without visco elastic insoles, using the mean peak plantar pressure as the parameter. Secondly it can be used in application related to athletic training for optimizing sports performance with thin-film pressure sensors and relatively inexpensive data acquisition hardware. More researches have been reported on athletic plantar pressure analysis in order to improve sports achievements, such as soccer balance training and forefoot loading during running.

Clinical gait analysis, the investigation of the pattern of walking, is the third application. Plantar pressure distribution was employed to detect gait patterns: normal gait, toe in, toe out, over supination, and heel walking gait abnormalities. Plantar pressure was applied in pathology like assessment of the diabetic foot. A variety of plantar pressure measurement systems are available in the market or in the research laboratories. With respect to their technical specifications and intended application, in general, there are two main types of measurement system should have the following features. devices: platform systems and in-shoe systems. Platform First, it should be wearable without intervening with the systems are usually embedded in a walkway. Howe ver, regular activity of the wearer. Secondly the sensing unit this kind of system is restricted to use in a laboratory or hospital, and used for barefoot measurements. In-shoe systems can be used to record the plantar pressure distributions within a shoe. Commercial products available from companies like Tekscan, Inc., capture dynamic in-shoe temporal and spatial pressure distributions, which were utilized for dynamic gait stability analysis, gait detection, and altered gait characteristics during running. Howe ver, those systems use electrical wires to connect in-shoe sensors and data acquisition system around the waist, which cause inconvenience and discomfort during strenuous exercises. A wireless structure shoe-integrated sensor system was de veloped for gait analysis and real-time feedback. In the system, hard de vices have been used cannot last for a long time because of fatigue of the sensing units. The data acquisition systems are often large and cannot be configured to connect with different remote receivers.

\section{HARDW ARE}

\section{A. Design Objectives}

With the purpose of collecting valid accurate data in natural condition of activities, the plantar pressure as the sensing units, which are not comfortable and 
has to perform with consistency for repeated testing. Thirdly

the

system

should

be convenient to wear. Usage of wireless technology to transfer lower limbs and whole body. In order to reduce the data would be the most convenient system to be established. system complexity, eight positions as indicated in fig 3 Taking into consideration the above requirements here we were selected at heel and metatarsal areas in the first present a pressure measurement and analysis technique prototype shoe, because these areas have higher employing wireless transmission. Design of a data pressure during normal activities of children, young, acquisition system and stable sensor network has been and old adults. Exact locations of sensors can be discussed. A software for setting the hardware parameter determined by depth shape of the subject's foot in soft and to monitor pressure values has been discussed.

\section{B. Sensor Selection} model. Earlier methods considered utilizing five sensors at the positions of heel, metatarsal, and hallux to achieve a clinical gait analysis. Another system was presented for a gait-phase detection system using three sensors at

Based on the aforementioned requirements, this underneath the heel and metatarsal areas. Six sensors at paper presents an in-shoe plant pressure measurement and the heel and metatarsal positions are adequate for such analysis system based on a fabric pressure sensing array. A clinical investigation in gait analysis. For sports and textile pressure sensing array whose internal structure as fitness, the Nike +iPod Sport Kit used just one sensor in been shown in fig 1(a) has been considered.

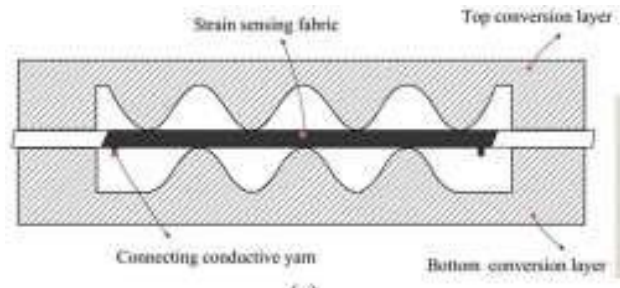

(a)

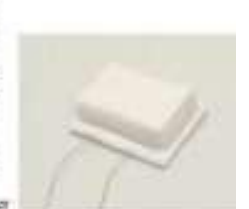

(b)

Fig. 1. Textile pressure sensor. (a) Sche matic diagra m of the structure of the press ure sensor (side view). (b) Package outlook.

It has a strain gauge factor of approximately 10 or above and excellent fatigue resistance (>100 000 cycles) for strain up to $40 \%$. The sensor measurement ranges are from $10 \mathrm{~Pa}$ to

$800 \mathrm{kPa}$, suitable for a wide variety of humanapparel

interfaces, such as loosely fit garment walking/running shoes etc. The sensor is packaged by silicon rubber so that moisture and dust will not affect its performance, as shown in Fig. 1(b).

Several textile pressure sensors are connected in an $-n+$ $1 \|$

line structure to construct a sensor array. One line is connected to each sensor as the $-1 \|$ ground line of the sensor array. Another line of each sensor is the output as the $-n$ thll signal line for the sensor array that contains sensors. By combination of several textile pressure sensors, the sensor array is able to measure the pressure on a single-point region, as well as the distributions.

\section{Pressure Points And Placement}

The pressure sensing area of the foot can be divided into 15 areas, as heel (area 1-3), midfoot (area 4-5), metatarsal (area

6-10), and toe (area 11-15), as shown in Fig. 2. These areas support most of the body weight and adjust the body balance. The measured force at these positions can be used to derive physiological, function information of the

the midfoot to measure the wearer's pace, distance, and energy consumption during running.

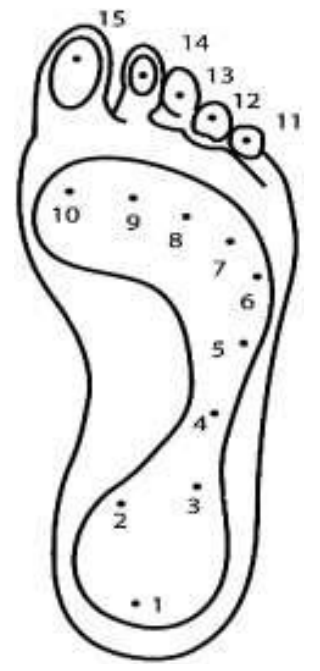

Fig 2. Various Pressure sensing Points

The Adidas-1 running shoe uses one sensor at the heel position to provide compression measurement for adjusting to running situation. Hence, six sensors are sufficient for sport or fitness assessments. Ideally,

adding additional sensors at

hallux and midfoot positions will enlarge the application scopes. As the textile sensor is relatively cheap and has a good fatigue resistance, it is easy to add more ensors in other applications.

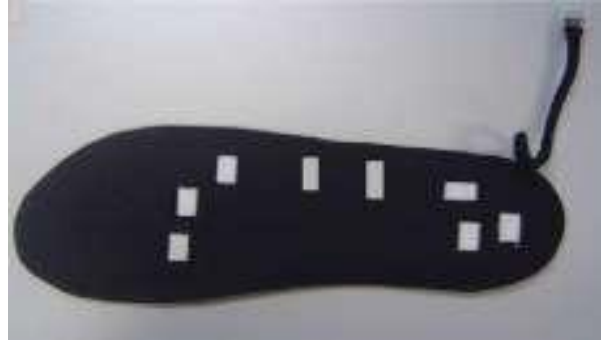

Fig. 3.Place ment of sensors 
In order to reduce the system complexity, eight positions (see Fig. 3) can be selected, because these areas have higher pressure during normal activities. A connector is attached to the sensors to transfer data to the circuit. Through the connector, voltage signals on the sensors are extracted and then sent to the embedded analog-todigital (A/D) converter channels in the processor. Finally, these values are wirelessly transmitted to a remote receiver by an antenna. The data acquisition system has the following advantages.

1) Small size and light weight.

2) Large transmission range.

3) Stable and repeatable performance.

4) Rechargeable battery configuration.

\section{Circuit Design} lustrates the typical implimentation requirments. The IS ideal for applications where miniaturization is a key selected position of sensors are wired to signal requirement. A blend of serial communications conditioning and amplification IC. A processor capable of interfaces ranging from a USB 2.0 Full Speed de vice, handling eight channels of analog values and which can multiple UARTS and on-chip SRAM of 8 kB upto 40 provide sufficient conversion accuracy is selected. An $\mathrm{kB}$, make these devices very well suited for ARM based LPC2148 has been selected for this purpose. communication gateways . Dual 10-bit ADC(s) with After processing the data it is given to the serial port and then conversion times as low as 2.44 microseconds per to the wireless transmitter.

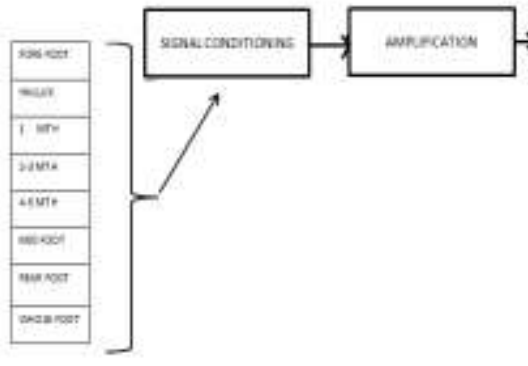

Fig 4. Design Esseentials

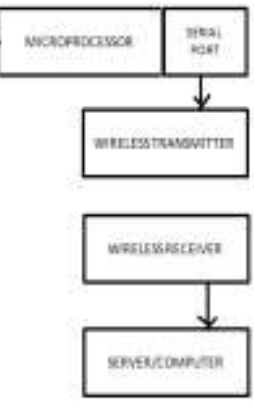

\section{E. Wireless Architecture}

Several radio protocols and open standards are now available, however, most of them do not support multiple sensors and are not compatible with low-power radio hardware. For this reason, we chose IEEE 802.15.4, which in recent years has emerged as the dominant wireless protocol for low-power sensor networks, and is also the physical-layer protocol for Zig bee . A wireless platform using Ultra Wide Band standard IEEE 802.15.4a is a possible alternative in the future. Using a star $1 \mathrm{~mW}(0 \mathrm{dBm})$, which provides a wireless detection topology for our network will minimize processing range of overhead and power consumption.

The data acquisition system is designed with small size antenna. and stable to power supply interference. There are Indoor range is significantly less and depends on the three possible ways to interpret the acquired data . building layout, but is approximately 15-20 $\mathrm{m}$ for the Bluetooth virtual serial port technology can be utilized to module with integrated antenna and 8-10m for the interface the circuit to mobile and PDAs. A Zig bee version with external antenna. These wireless operating receiver and a laptop environment can increase the distances are sufficient for our current health and medical coverage area of the system so that the subject can be research needs. 
IJIREEICE

International Journal of Innovative Research in

Electrical, Electronics, Instrumentation and Control Engineering

ISO 3297:2007 Certified

Vol. 5, Issue 3, March 2017

\section{Radio Base Station}

A popular method that is used to collect data from multiple radio modules and sensors is universal serial bus (USB), which has a USB interface to plug into PC's and laptops. For software development a platform of OS which is easier to use has been adopted. Accordingly Windows is the platform used to develop the front end. Fig 5 shows the means by which an environment for software development has been utilized. The software is designed is such a manner that it is capable of providing secured entry to use the software using authentication. It is also possible to set the various sensing points that are to be added for data collection. Also provision had been made to set the serial data rates and other parameter by front end module. The hardware setup placed inside the sole wirelessly transmits the pressure values to the receiving module connected to the serial port. This information is store in a database continuously. Further processing is done by the front end software.

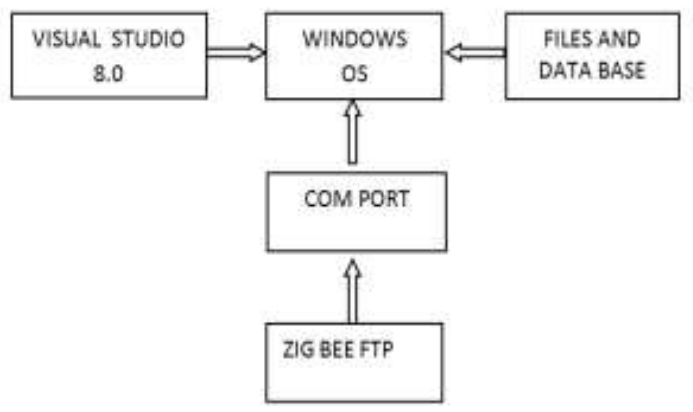

FIG 5. Softwa re development.

Upon start up various configurations are made. These settings are transferred through serial port to the wireless module. This in turn sends these setting to the remote hardware. Upon successful configuration the start data collection process is initiated. Wherein the data from the hardware is received wirelessly. These data are stored in a buffer after which the system waits for the next data to arrive. This sequence is repeated until a stop signal is given from the software.

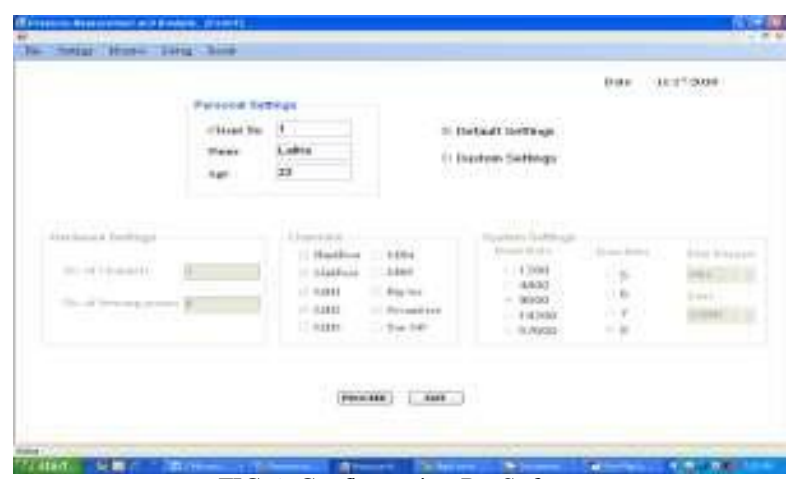

FIG 6. Configurat ion By Software

Fig 6 shows the actual settings window where personal details sensor point settings and other system settings are done in software to configure hardware.

The data thus received is used for various calculations.

$$
\begin{aligned}
& \text { Mean }=\operatorname{Sum}(P 1, \ldots P i, \ldots P n) / n \\
& \text { Peak }=\operatorname{Max}(P 1, \ldots P i, \ldots P n)
\end{aligned}
$$

\section{RESULTS}

Results have been plotted as real time graphical displays and the peak and mean pressure are also displayed. A pie chart showing the distribution of pressure has also been given. Fig 7 shows pressure distribution at various sensing points. This gives an idea about where the maximum pressure that was recorded during the entire record time. A real time signal display window shows the values as recording is done which helps in monitoring instantaneous real time pressure values.

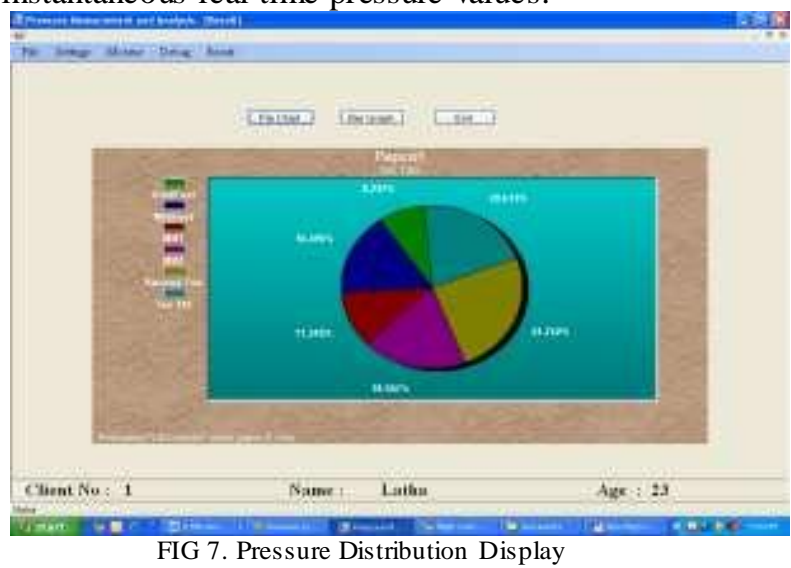

Fig 8 shows a sample recorded output recording from software as signal variations. We can start, stop recording and store the recorded values to be viewed later for analysis.

FIG 8. Rea1 Time Display

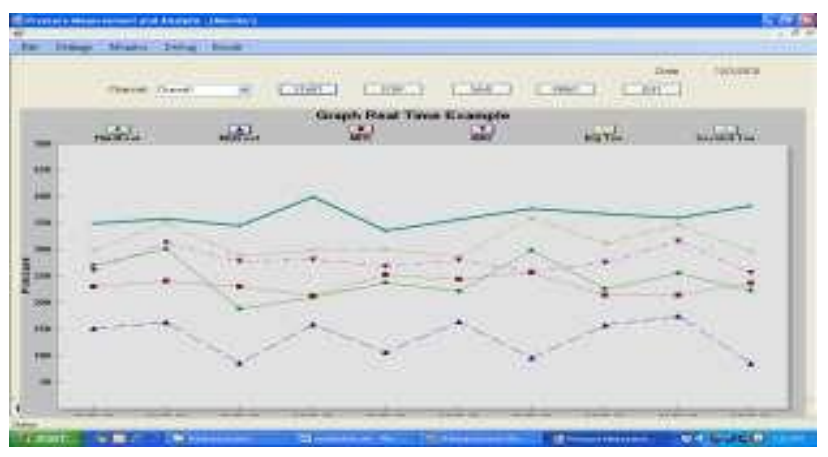

\section{CONCLUSION AND FUTURE WORK}

As demonstrated in this paper, recent advances in lowpower radio electronics and wireless protocols are enabling the development of new technology for longterm, comfortable sensing of pressure information in new areas of health and medical research. New wearable materials, coupled with small long lasting batteries, now provide the means to collect data over much longer time scales and in nonclinical settings, and the means for 
individuals to control the collection and communication of data by easily putting on or taking off the sensor (not needing the help of a researcher, and not having data sensed from them if they do not want to be sensed). It have been shown data and evaluations in this paper to indicate that these new sensors, while non traditional in their placement and design, are capable of gathering data comparable to data gathered with traditional sofisticated sensors . Thus, the system we have de veloped provide an important contribution over existing systems for gathering data in long-term naturalistic settings. It is our goal to help make lightweight portable sensor platforms such as the ones presented here accessible to a wider number of researchers and to indi viduals who wish to have help understanding and communicating their foot pressure changes. We envision that the strong connection between affective computing and health will also lead to new forms of understanding, diagnosing, and supporting the growing number of people interested in footwear evaluation, athletic training, clinical gait analysis, and pathology foot diagnosis. Research on the area of developing a stand alone hand held de vice to the entire monitor the above method would be considerable development. Also the is scope to transfer the recorded data through internet for data analysis to experts in any location and give feedback.

\section{REFERENCES}

[1] L. A. Lavery, S. A. Vela, J. G. Fle ishli, D. G. Arms trong, and D. C. Lavery, - Reducing plantar pres s ure in the neuropathic foot-A comparis on of footwear,\| Diabetes Care, vol. 20, no. 11, pp. 1706-1710, 1997.

[2] S. J.Morris and J. A. Pa radis o, -Shoe-integrated s ens or sys tem for wire les s gait analys is and rea l-time feedback, $\|$ in Proc. 24th Annu. Conf. Annu. Fall Meet. Biomed. Eng. Soc. EMBS/BMES Conf., Hous ton, 2002, pp. 2468- 2469. [3] Q. , H. Wei, W. , L. Lu, Z.Y. Fu, and S. B. Lu, -A new s ys tem for foot press ure meas urement and gait analys is, $\|$ Chin. J. Biomed. Eng., vol. 19, no. 1, pp. 32-40, 2000.

[4] E. Kellis, -Plantar pressure dis tribution during barefoot standing, walking and landing in pres chool boys ,\| Gait Posture, vol. 14, no. 2, pp. 92-97, 2001. [5] M. , J. Hes sert, M. Vyas, J. Leach, K. Hu, L. , A. Lips it $\mathrm{z}$, and V. Novak, -Foot press ure dis tribution during walking in young and old adults, $\| B M C$ Geriatr., vol. 5, p. 8, 2005.

[6] S. Ba mberg, A. Y. Benbas at, D. M. Scarborough, D. E. Krebs, and J. A. Paradis o, - Gait analys is using a shoe-integrated wire les s sens or s ys tem,\| IEEE Trans. Inf. Technol. Biomed., vol. 12 , no. 4, pp. 413-423, Ju 1. 2008 